\title{
Development of an intervention system for linkage-to-care and follow-up for hepatitis $B$ and $C$ virus carriers
}

\author{
Minami Kikuchi ${ }^{1,2} \cdot$ Motoji Sawabe $^{2} \cdot$ Haruyo Aoyagi $^{1} \cdot$ Kosho Wakae $^{1} \cdot$ Koichi Watashi $^{1,3} \cdot$ Satoru Hattori ${ }^{4}$. \\ Naoto Kawabe ${ }^{5} \cdot$ Kentaro Yoshioka $^{6} \cdot$ Junko Tanaka ${ }^{7} \cdot$ Masamichi Muramatsu $^{1} \cdot$ Takaji Wakita $^{1} \cdot$ Hideki Aizaki $^{1}$ (1)
}

Received: 29 June 2021 / Accepted: 26 October 2021 / Published online: 2 December 2021

(c) Asian Pacific Association for the Study of the Liver 2021

\begin{abstract}
Background Poor connections in the cascade of viral hepatitis care have been discussed around the world. In 2011 in Japan, 500,000 to 1.25 million hepatitis B and C virus carriers needed to consult with hepatologists, so linkage-to-care (LTC) needs to be promoted. Therefore, in this study, to improve LTC and care-seeking behaviors, we attempted to establish a communitybased intervention system and evaluate its effectiveness by analyzing behavior modifications.

Methods In a model city, Okazaki (population: 387,887 as of 2019), LTC was encouraged among HBV and HCV carriers by annually mailed brochures, and their care-seeking behaviors were followed up through questionnaires for 8 years (2012-2019). Their behavior modifications and demographic characteristics were analyzed anonymously in cooperation with community health workers, hepatologists, and researchers.

Results Through regional HBsAg and anti-HCV screening, $333 \mathrm{HBV}$ and $208 \mathrm{HCV}$ carriers were identified. Before the intervention, only $34.7 \%$ (25/72) of HBV- and 34.3\% (24/70) of HCV-positive individuals had consulted with hepatologists. However, in 2019, after the intervention, these proportions increased to $79.8 \%$ (91/114) and 91.2\% (52/57), respectively. Access to outpatient care and treatment uptake also continuously improved. However, individuals over 70 years of age were
\end{abstract}

Hideki Aizaki

aizaki@nih.go.jp

Minami Kikuchi

mi-kikuchi@niid.go.jp

Motoji Sawabe

m.sawabe.mp@tmd.ac.jp

Haruyo Aoyagi

aoyagi@nih.go.jp

Kosho Wakae

wakae@nih.go.jp

Koichi Watashi

kwatashi@nih.go.jp

Satoru Hattori

hokenzoshin@city.okazaki.lg.jp

Naoto Kawabe

kawabe@fujita-hu.ac.jp

Kentaro Yoshioka

kyoshiok@fujita-hu.ac.jp

Junko Tanaka

jun-tanaka@hiroshima-u.ac.jp

Masamichi Muramatsu

muramatsu@nih.go.jp
Takaji Wakita

wakita@nih.go.jp

1 Department of Virology II, National Institute of Infectious Diseases, 1-23-1 Toyama, Shinjuku, Tokyo 162-8640, Japan

2 Department of Molecular Pathology, Graduate School of Medical and Dental Sciences, Tokyo Medical and Dental University, 1-5-45 Yushima, Bunkyo, Tokyo 113-8519, Japan

3 Research Center for Drug and Vaccine Development, National Institute of Infectious Diseases, 1-23-1 Toyama, Shinjuku, Tokyo 162-8640, Japan

4 Okazaki City Public Health Center, 2-1-1 Wakamiya, Okazaki, Aichi 444-8545, Japan

5 Department of Gastroenterology and Hepatology, School of Medicine, Fujita Health University, 1-98 Dengakugakubo, Kutsukake, Toyoake, Aichi 470-1192, Japan

6 Center for Liver Diseases, Meijo Hospital, 1-3-1 Sannomaru, Naka, Nagoya, Aichi 460-0001, Japan

7 Department of Epidemiology, Infectious Disease Control and Prevention, Graduate School of Biomedical and Health Sciences, Hiroshima University, 1-2-3 Kasumi, Minami, Hiroshima, Hiroshima 734-8551, Japan 
significantly less likely to engage in care-seeking behaviors $(p<0.05)$, and significantly fewer HCV-positive females received treatment $(p=0.03)$.

Conclusions A paper-based reiterative intervention encouraging LTC and follow-up successfully improved the care-seeking behaviors of hepatitis virus-positive individuals and enabled their behavior modifications to be monitored. Further trials are required to advance the system by age- and gender-specific interventions.

\section{Graphical Abstract}

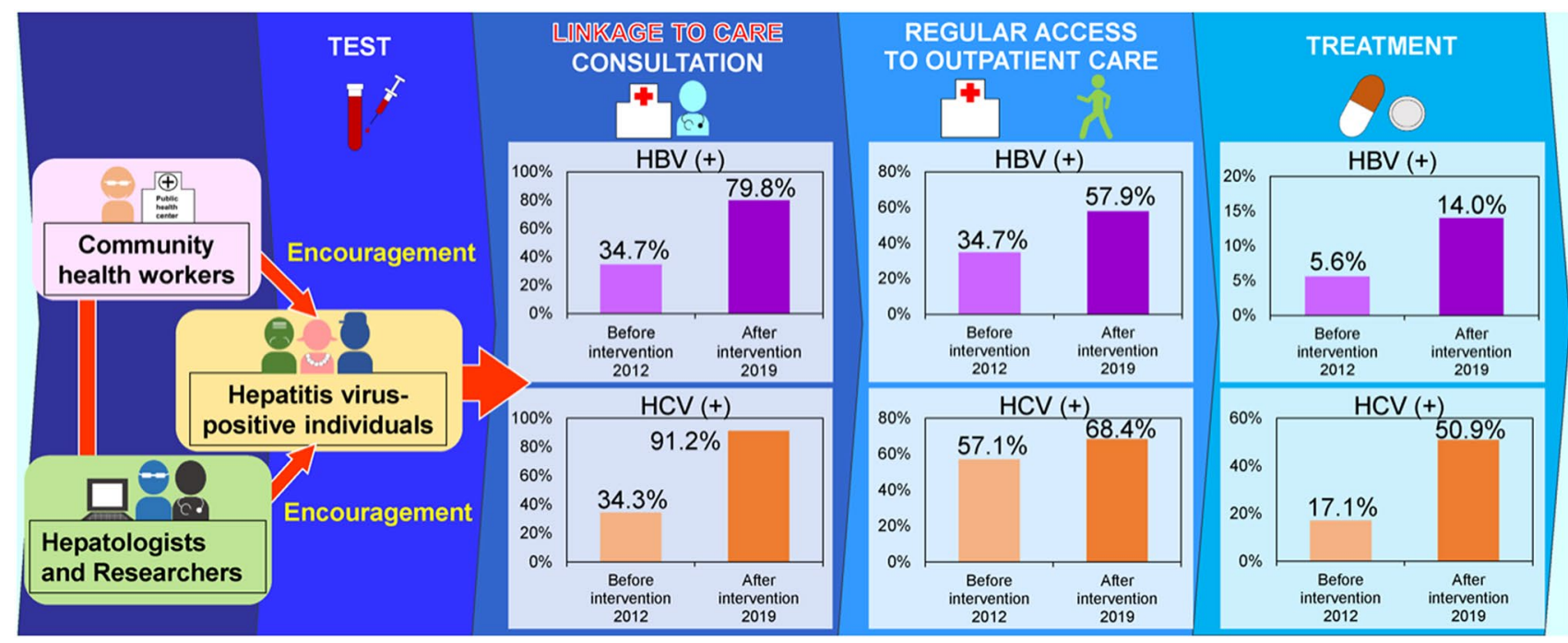

Keywords Viral hepatitis · HBV · HCV · Care continuum · Cascade of care $\cdot$ Care-seeking behavior $\cdot$ Consultation · Access to outpatient care $\cdot$ Treatment uptake $\cdot$ Hepatitis enlightenment activity

\section{Introduction}

According to the World Health Organization (WHO), in 2015 , more than 300 million people worldwide were living with hepatitis B virus (HBV) or hepatitis C virus (HCV) [1]. For viral hepatitis elimination, the WHO advocated the necessity of improving the "continuum of viral hepatitis services", which includes testing, linkage-to-care (LTC), treatment and long-term follow-up as chronic care. The WHO recommends that $90 \%$ of hepatitis virus carriers should be diagnosed, and that $80 \%$ should be treated by $2030[1,2]$. However, in reality, the WHO estimated that only approximately $9 \%$ of HBV and $20 \%$ of HCV carriers have been diagnosed, and further, that only $8 \%$ and $7 \%$ of those, respectively, have been treated [1]. Therefore, the global expansion of interventions is considered an urgent issue [1].

Meanwhile, in Japan, in 2011, the number of HBV and HCV carriers was estimated to be between 2.09 and 2.84 million [3]. Of these carriers, approximately 780,000 individuals have not been diagnosed, and 500,000 to 1.25 million have been diagnosed but have not consulted with doctors yet, while approximately 810,000 have already been treated in hospitals. Thus, in Japan, the number of unconsulted carriers exceeds that of undiagnosed carriers [3,4]. The number of undiagnosed hepatitis virus carriers was successfully reduced [3] because the Japanese government launched national hepatitis virus screening in 2002 [4], and the Basic Act on Hepatitis Measures has been in force since 2010 [5]. On the other hand, the promotion of LTC and longterm follow-up has been more challenging. Even though Japanese government has already established specific countermeasures for reported major impediments of LTC, such as expensive medical costs, inconvenient access to health-care institutions, and cultural and linguistic differences [6, 7], based on the Basic Act [5], Japan is still burdened with a large number of unconsulted carriers.

In addition, reports on systematic care for hepatitis viruspositive individuals are quite limited. Most challenges have been seen in high-income countries and targeted high-risk populations, such as immigrants or people who inject drugs [7-9]. Moreover, retrospective studies on the care-seeking behaviors of hepatitis virus-positive individuals or trials involving longterm follow-up remain scarce [10-13] because the effectiveness of interventions has mostly been evaluated within a few years after $\mathrm{HBV}$ or HCV mass-screening campaigns.

Therefore, in 2012, a continuous intervention system for encouraging LTC and long-term follow-up for hepatitis viruspositive individuals was established as a model project through cooperation between the National Institute of Infectious Diseases (NIID), a regional core center for treating liver diseases, 
and a public health center. This study aimed to elucidate the modifications in behavior among hepatitis virus-positive individuals and the association between their care-seeking behaviors and demographic characteristics in the model project to evaluate the effectiveness of continuous interventions for improving LTC and long-term follow-up, anticipating the expanding application of this system throughout Japan in the future.

\section{Materials and methods}

\section{Hepatitis virus screening and study participants in the model city}

Okazaki city (population: 387,887 as of April 2019) in Aichi Prefecture was selected as a model city. The details of
Okazaki city including its residential area classification are provided in Supplementary Fig. S1.

The details of the implementation scheme of hepatitis virus screening and the criteria of study participants, HBsAg- or antiHCV antibody-positive individuals identified during 2008-2018, are described in Supplementary Method 1.

\section{Continuous intervention system for encouraging LTC and follow-up in the model city}

The continuous intervention system for encouraging LTC and follow-up was established through cooperation with the NIID, a regional core center in Aichi Prefecture, and a public health center in Okazaki city. The scheme of the paper-based intervention system is shown in Fig. 1. The flow of the system and contents of documents sent to hepatitis virus-positive individuals are described in Supplementary Method 2. The intervention was implemented annually for

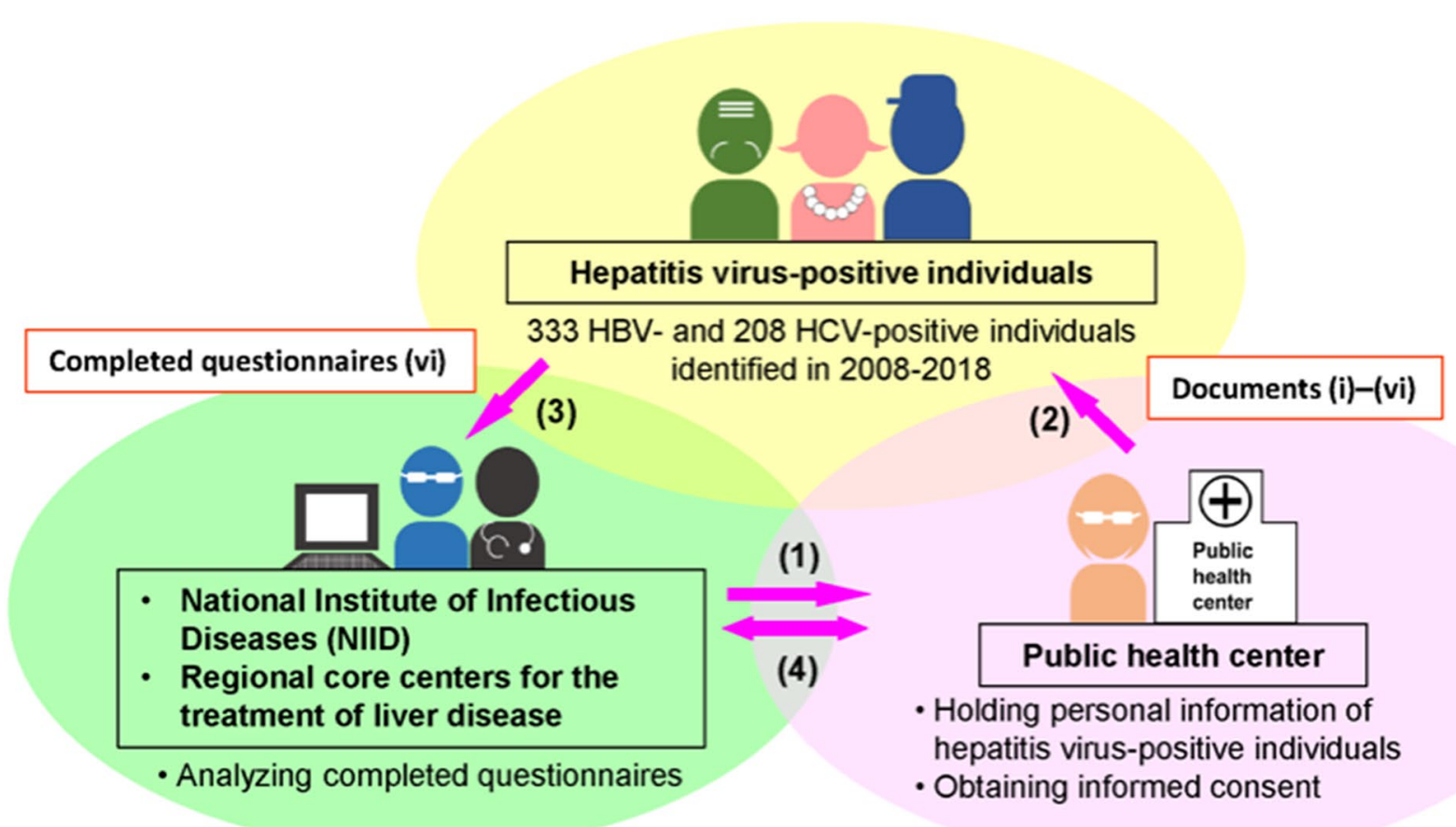

Fig. 1 Scheme of the intervention system for linkage-to-care (LTC) and follow-up for hepatitis virus-positive individuals. The intervention system was established through cooperation between the National Institute of Infectious Diseases (NIID), a regional core center for the treatment of liver disease, and a public health center. (1) Prepare and send documents (i) through (v) encouraging LTC and questionnaires (vi) to the public health center. (2) Attach addresses and send all documents (i) through (vi) to hepatitis virus-positive individuals. (3) Read documents (i) through (v) encouraging LTC and send completed questionnaires (vi) to the NIID. (4) Analyze the questionnaire responses and share the results with the public health center 
8 years (2012-2019) and LTC and follow-up were encouraged repeatedly among hepatitis virus-positive individuals.

\section{Questionnaire}

The process of formulating questionnaire is referred in Supplementary Method 3 and the questions and answer choices are shown in Supplementary Table S1.

\section{Preliminary investigation}

In 2012, to evaluate the effectiveness of the continuous intervention, a preliminary investigation was implemented on $159 \mathrm{HBV}$ - and $153 \mathrm{HCV}$-positive individuals detected through the screening during 2008-2012. The results from the pre-investigation were regarded as the non-intervention control.

After the intervention system was fully established, those $159 \mathrm{HBV}$ - and $153 \mathrm{HCV}$-positive individuals (312 individuals) were included in the system, LTC was encouraged by documents, and they responded to the questionnaire (Supplementary Table S1) in the same year, considering ethical aspects. Next, the intervention was implemented as described until 2019. Since the hepatitis virus-screening and intervention were all implemented in Okazaki city accordingly, and those 312 individuals were fully included in the intervention system, the demographic characteristics of those 312 individuals and hepatitis virus-positive individuals who were identified after the pre-investigation were considered to be the same, and the non-intervention and intervention cohorts were balanced to assess outcomes in this study.

\section{Data assembly and statistical analysis}

We used chi-squared and Fisher's exact tests to analyze the associations between demographic characteristics of hepatitis virus-positive individuals, such as age (40-59, 60-69, and above 70 years old), gender (male and female), and residential area (urban, suburban, and rural areas), and the number of individuals who had a consultation, access to outpatient care and treatment, the frequencies of these careseeking behaviors, and the reasons why they did or did not engage in the behaviors. Logistic regression analysis using age, gender, and residential area was performed to identify the factors related to four outcomes: response to the questionnaire, consultation, regular access to outpatient care, and treatment. We also performed interrupted time series (ITS) analysis [14], a type of multiple linear regression model, to evaluate the intervention. The details of these analyses are provided in Supplementary Method 4. $p$ values $<0.05$ were considered statistically significant.

\section{Results}

\section{Demographics of the hepatitis virus-positive individuals and dropouts}

In Okazaki city, free hepatitis virus testing was performed among citizens aged 40 years and over who had no history of hepatitis virus screening and wished to be examined. In total, 35,910 and 35,888 citizens had undergone HBV and HCV screening, respectively, during 2012-2018. The mean HBV- and HCV-positive rates were $0.52 \%$ and $0.26 \%$, respectively, over these 7 years (Fig. 2a). The demographic characteristics of the hepatitis virus-positive individuals are shown in Supplementary Table S2. The HBV-positive individuals consisted of 165 males (mean age, 62.5 years) and 161 females (mean age, 60.1 years); the age and gender of 7 individuals were unknown. On the other hand, the HCV-positive individuals consisted of 93 males (mean age, 64.2 years) and 102 females (mean age, 71.2 years); the age and gender of 13 individuals were unknown. Of these hepatitis virus-positive individuals, three males and one female were co-infected with HBV and HCV. For both HBV and HCV infections, the number of positive individuals tended to decrease; however, the proportions of individuals over 70 years of age increased from 2008 (Fig. 2b, c). In the hepatitis virus screening, the total number of screening recipients in their $40 \mathrm{~s}$ and $60 \mathrm{~s}$ was larger than that for other generations in 2012-2019 (Supplementary Figs. S2a and S2b). The HBV-positive rate was highest among screening recipients who were in their $50 \mathrm{~s}$ (Supplementary Fig. S2a). By contrast, the HCV-positive rate increased with age and was especially high among females over 80 years of age (Supplementary Fig. S2b). Regarding residential areas, 185 (35.9\%) hepatitis virus-positive individuals lived in an urban area, $208(40.3 \%)$ in the suburbs, and $123(23.8 \%)$ in a rural area (Supplementary Table S2) (Supplementary Fig. S1). In total, 53 (15.9\%) HBV- and 50 (24.0\%) HCVpositive individuals who participated in the study moved out of the study area or died during the follow-up period (2012-2019).

\section{Behavior modifications of hepatitis virus-positive individuals}

The annual investigation and LTC encouragement for hepatitis virus-positive individuals were implemented by mailing letters and brochures through cooperation between the NIID, a regional core center, and a public health center for 8 years (Fig. 1). The participants' care-seeking behaviors were analyzed during 2012-2019, and their behavior 
Fig. 2 Hepatitis virus-positive rates and the numbers of hepatitis virus screening recipients and positive individuals in Okazaki city. a Hepatitis virus-positive rates and the number of screening recipients by year. HBV- and HCV-positive rates are shown by solid and dashed lines, respectively. Dark and light gray bars show HBV and HCV screening recipients, respectively. b, c Number of hepatitis virus-positive individuals by age group and year. Patterns in the graphs show age groups of individuals aged $40-90$ years. Solid lines show the proportions of individuals aged over 70 years. b represents HBV and c $\mathrm{HCV}$ infections

modifications were monitored anonymously based on their answers in the questionnaires using identification numbers provided by the public health center from 2013 to 2019 as a follow-up. The mean response rate for the questionnaires was $46.5 \%$ for HBV-positive and $44.1 \%$ for $\mathrm{HCV}$-positive individuals in 2012-2019, which was comparable to that in the preliminary investigation in 2012 (Supplementary Table S3). As a result, 72.7\% (242/333) of HBV-positive individuals and $68.3 \%$ (142/208) of HCV-positive individuals responded to the questionnaires at least once over 7 years (2013-2019). In the pre-investigation, the proportions of $\mathrm{HBV}$ - and $\mathrm{HCV}$-positive individuals who consulted a hepatologist were $34.7 \%(25 / 72)$ and $34.3 \%$ (24/70), respectively. These proportions significantly increased over time after the intervention: $79.8 \%(91 / 114)$ of HBV- and $91.2 \%$ (52/57) of HCV-positive individuals consulted a hepatologist in 2019 (Fig. 3a). The results of the ITS analysis describing the trend for the proportion of individuals who have a consultation is shown in Supplementary Fig. S3a, S3b and S3c. As for the "level change", "Consultation-HBV" ( $p=0.0003)$ (Supplementary Fig. S3a, S3c) and "Consultation-HCV" ( $p<0.0001)$ (Supplementary Fig. S3b, S3c) were significantly increased after the intervention. Moreover, as for the "slope during the intervention", "Consultation-HBV" ( $p=0.0336)$ (Supplementary Fig. S3a, S3c) and "Consultation-HCV" $(p=0.0004)$ (Supplementary Fig. S3b, S3c) also exhibited significantly positive trends during the intervention. The cumulative numbers of individuals who reported consulting with a hepatologist were 58.6\% (195/333) HBV- and $62.0 \%$ (129/208) HCV-positive individuals (Fig. 3b). In other words, approximately $60 \%$ of the individuals consulted a hepatologist at least once until 2019. Moreover, among newly detected hepatitis virus-positive individuals, $67.0 \%$ (65/97) of HBV- and $67.4 \%$ (31/46) of HCVpositive individuals consulted a hepatologist within 1 year after the first intervention for encouraging LTC (Fig. 3c). More than $50 \%$ of the reasons for not consulting a hepatologist among the HBV- and HCV-positive individuals was "No symptoms" (Fig. 3d).

The proportions of HBV- and HCV-positive individuals who regularly accessed outpatient care in the preinvestigation were $34.7 \%(25 / 72)$ and $57.1 \%(40 / 70)$, a

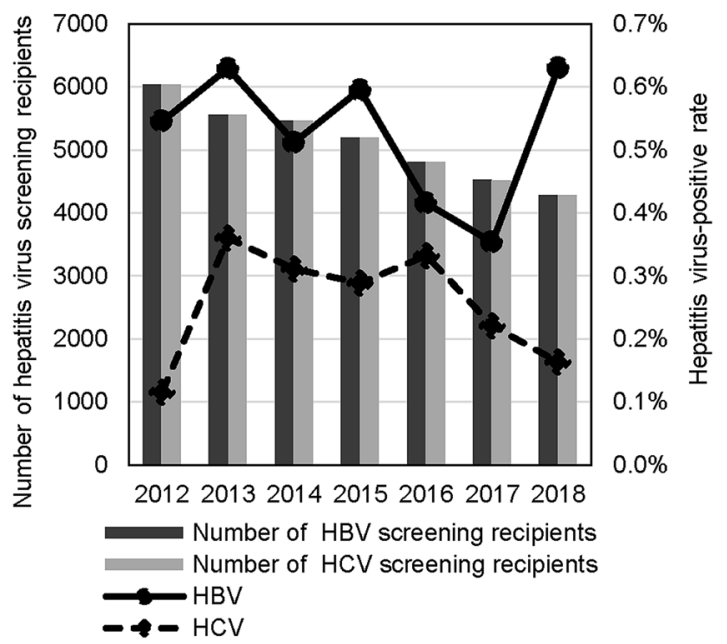

b

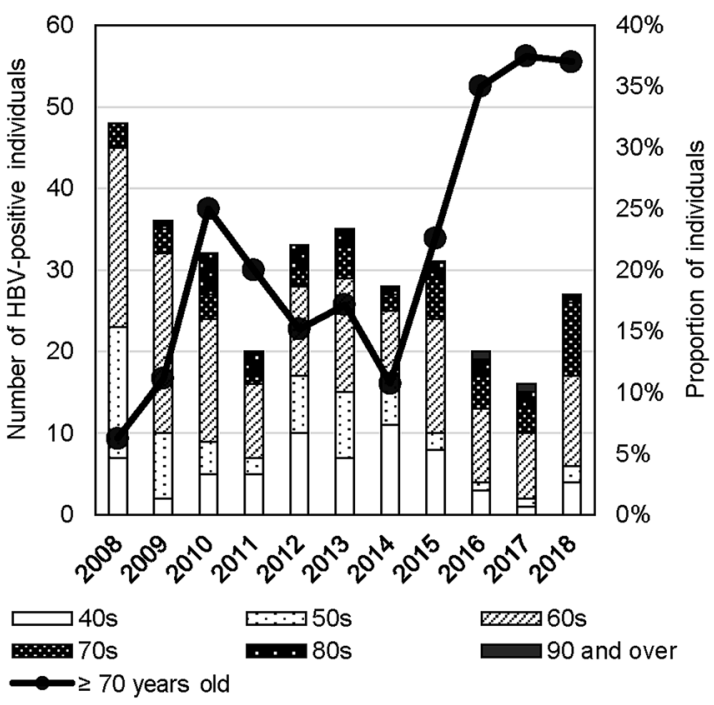

C

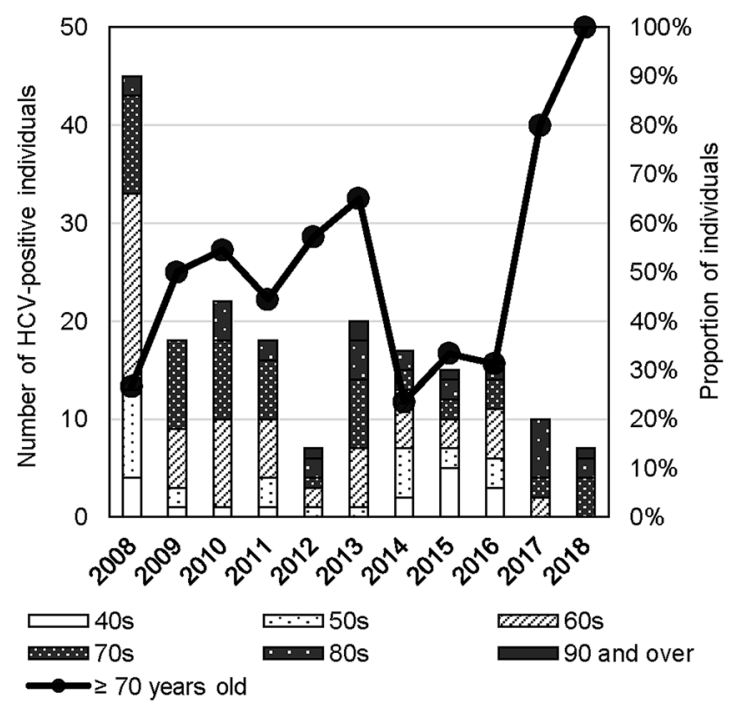


a

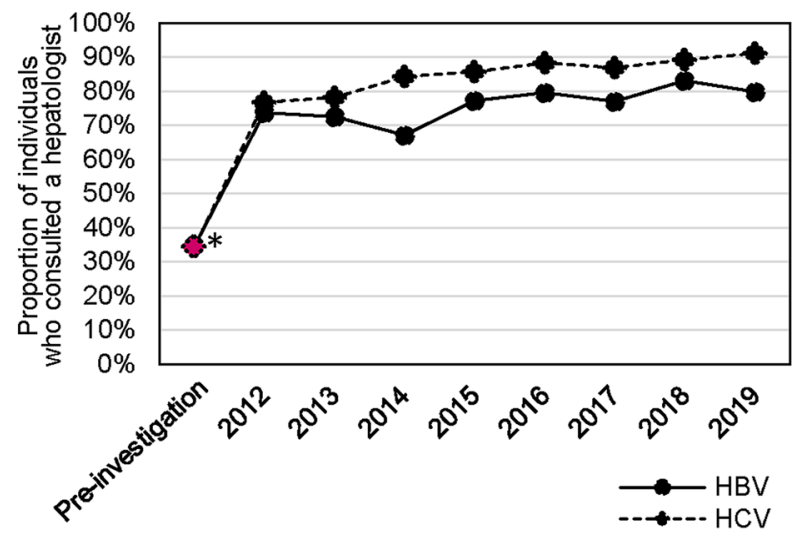

Number of respondents
HBV
(72) (72)
(102) $(79)$
(114) (122) (126)
(118) (114)
$\mathrm{HCV}$
(70) (69) (78)
(38)
(77) $(68)$
(68) (65)
$(57)$

C

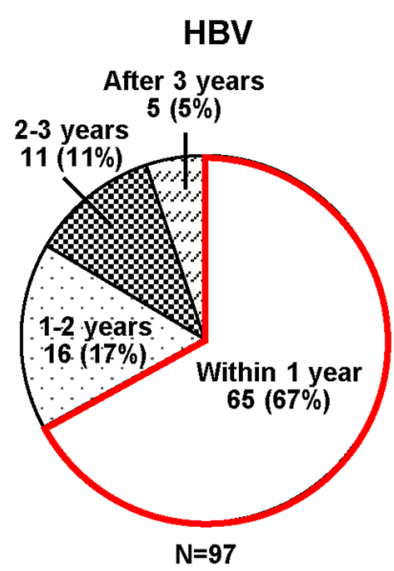

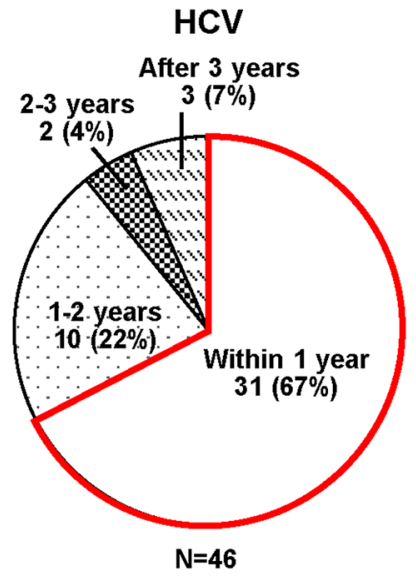

Fig. 3 Behavior of seeking consultations. a The proportion of hepatitis virus-positive individuals who consulted a hepatologist during 2012-2019. The solid line represents HBV and the dashed line represents HCV infections. The asterisks show the results in the pre-investigation. b Cumulative number of hepatitis virus-positive individuals who consulted a hepatologist during 2013-2019. c Interval between

respectively; these proportions also increased after the start of the intervention (Fig. 4a): $57.9 \%$ (66/114) and $68.4 \%$ (39/57) accessed outpatient care regularly in 2019, respectively. There was no significant increase regarding the "level change" in both HBV and HCV infection, while as for the "slope during the intervention", "Access to outpatient care-HBV" ( $p=0.0107)$ (Supplementary Fig. S3a, S3c) showed significant positive trends. Among the HBVand $\mathrm{HCV}$-positive individuals who consulted a hepatologist, $48.9 \%$ (70/143) and 67.8\% (61/90), respectively, regularly accessed outpatient care (Fig. 4b, left). Furthermore, of these individuals, $65.7 \%$ (46/70) and $49.2 \%$ (30/61), b

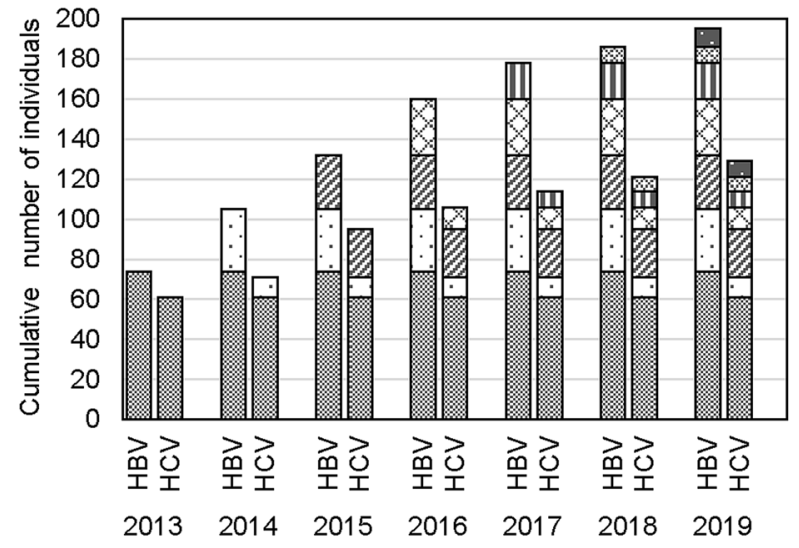

Consulted in 2013 Consulted in 2014 CConsulted in 2015 BConsulted in 2016 mConsulted in 2017 BConsulted in 2018

Consulted in 2019

d

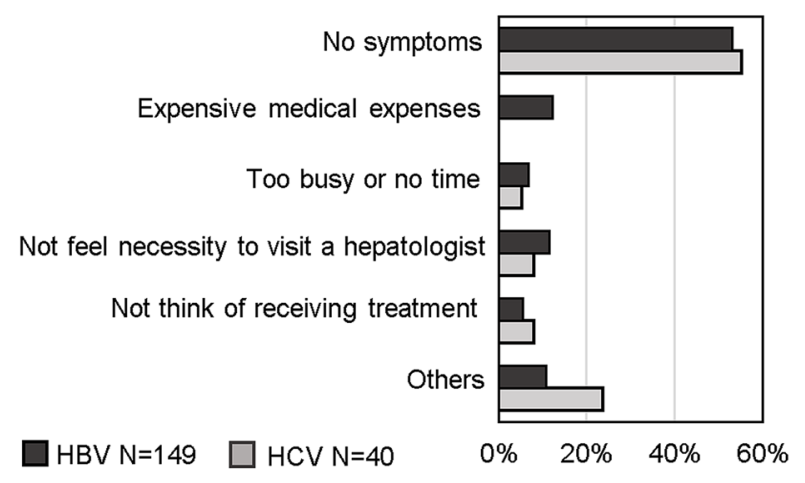

the first encouragement of LTC and the first consultation with a hepatologist. The red frames illustrate that approximately $70 \%$ of both HBV- and HCV-positive individuals consulted a hepatologist within 1 year after the first intervention for encouraging LTC. d Reasons for not consulting a hepatologist (multiple answers were allowed)

respectively, continued accessing outpatient care for more than 4 years (Fig. 4b, right). Moreover, of these 76 individuals (46 HBV- and $30 \mathrm{HCV}$-positive individuals), $46.1 \%$ (35/76) never stopped accessing outpatient care, whereas $36.8 \%$ (28/76) restarted access after it had been stopped. "No symptoms" and "My physician did not recommend regular access to outpatient care" were the major reasons why HBV- and HCV-positive individuals did not access outpatient care on a regular basis (Fig. 4c). Especially among HCV-positive individuals, "Cured by previous treatments" was the most common reason. 
Fig. 4 Behavior of regular access to outpatient care. a The proportion of hepatitis virus-positive individuals who regularly accessed outpatient care during 2012-2019. The solid line represents HBV and the dashed line represents HCV infections. The asterisks show the results in the pre-investigation. b The pie charts on the left show the proportion of individuals who accessed outpatient care, and those on the right show the duration of access. The red frames illustrate that over $65 \%$ of $\mathrm{HBV}$ - and approximately $50 \%$ of $\mathrm{HCV}$-positive individuals continued accessing outpatient care for more than 4 years. $\mathbf{c}$ Reasons for not accessing outpatient care (multiple answers were allowed)

The proportions of HBV- and HCV-positive individuals who received treatment in the pre-investigation were $5.6 \%(4 / 72)$ and $17.1 \%$ (12/70), respectively; however, these proportions also increased after the intervention: $14.0 \%(16 / 114)$ and $50.9 \%(29 / 57)$ received treatment in 2019, respectively (Fig. 5a), and a significant increase was observed in the "level change" in "Treatment-HCV" $(p=0.0304)$ (Supplementary Fig. S3b, S3c). "My hepatologist recommended treatment" was the most common reason for receiving treatment among both HBV- and HCV-positive individuals, followed by "Feel the necessity to receive treatment" and "Afraid of disease progression" (Fig. 5b).

\section{Demographic characteristics influencing the care-seeking behaviors of hepatitis virus-positive individuals}

The relation between the hepatitis virus-positive individuals' demographic characteristics and care-seeking behaviors was analyzed to elucidate whether demographic characteristics influenced care-seeking behaviors. For the care-seeking behaviors, the following questions were analyzed: (I) whether hepatitis virus-positive individuals engaged in careseeking behaviors during 2013-2019, (II) how often hepatitis virus-positive individuals consulted a hepatologist, and (III) why hepatitis virus-positive individuals did not consult a hepatologist, access outpatient care regularly, or received treatments (Supplementary Table S1).

\section{Whether hepatitis virus-positive individuals engaged in care-seeking behaviors during 2013-2019}

The proportions and adjusted odds ratios were low for the questionnaire responses ( $p=0.0009$ for $40-59$ years vs. over 70 and $p=0.0002$ for $60-69$ years vs. over 70 , respectively), consultations ( $p=0.0039$ and $p<0.0001$, respectively), and regular access to outpatient care ( $p=$ 0.0435 and $p=0.0147$, respectively) for HBV-positive individuals who were over 70 years of age compared with those who were 40-59 and 60-69 years old (Table 1). They were also low for older HCV-positive individuals (above 70 years of age) compared with younger generations (40-59 and 60-69 years); for the questionnaire a

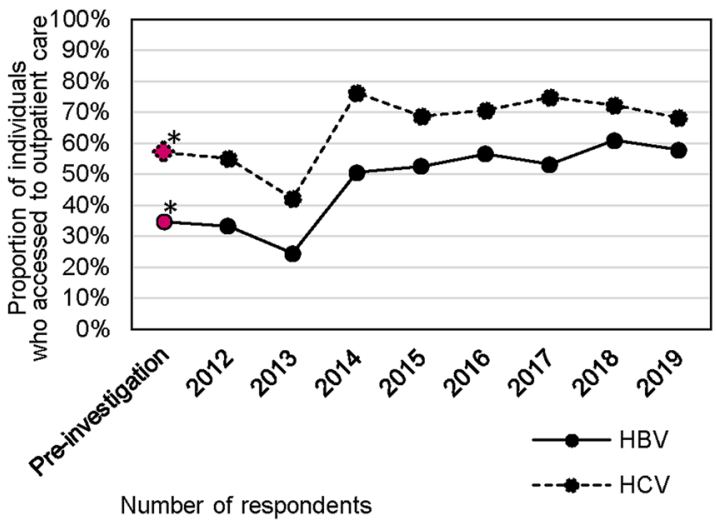

HBV (72) (72) (102) (79) (114) (122) (126) (118) (114)

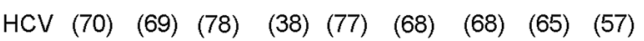

b
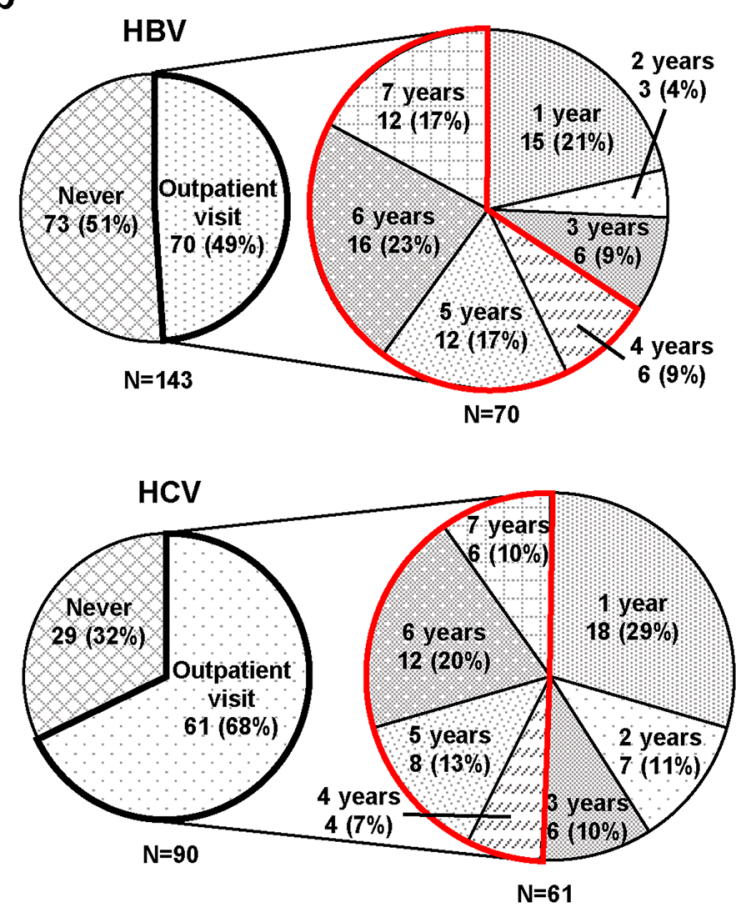

C

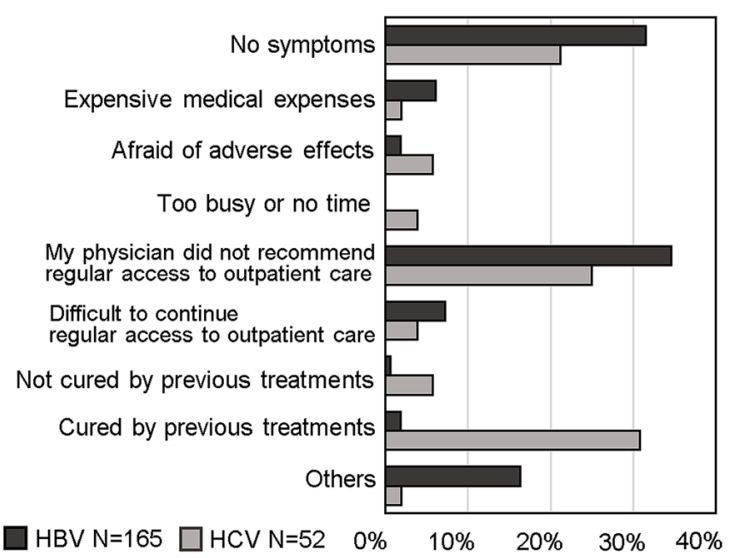


a

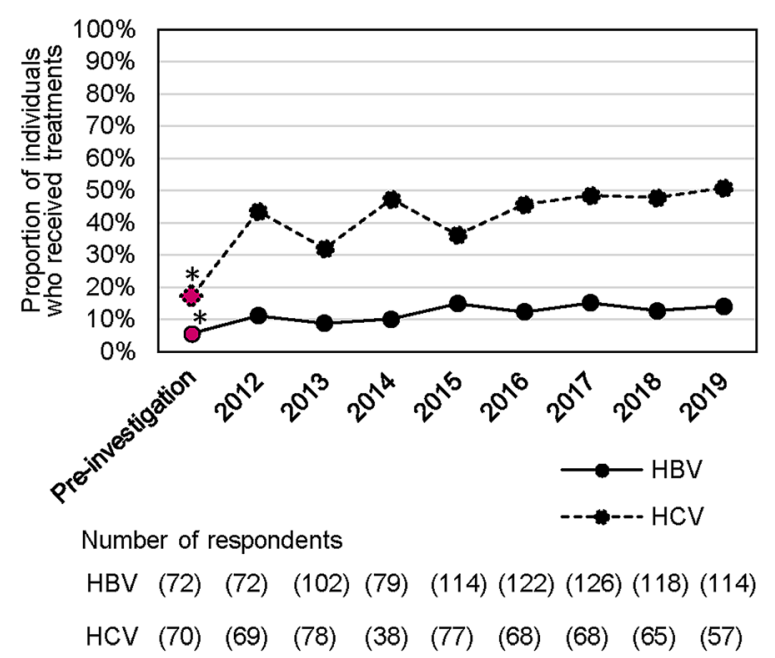

b

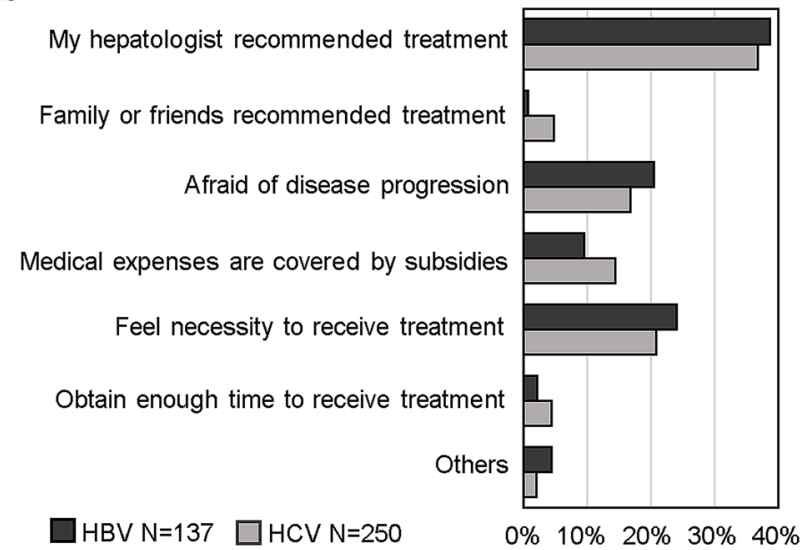

Fig. 5 Behavior of receiving treatment. a The proportion of hepatitis virus-positive individuals who received treatment during 2012-2019. The solid line represents HBV and the dashed line represents HCV infections. The asterisks show the results in the pre-investigation. b Reasons for receiving treatment (multiple answers were allowed)

responses ( $p=0.0503$ for $40-59$ years vs. over 70, and $p$ $=0.0454$ for $60-69$ years vs. over 70 , respectively), consultations ( $p=0.0203$ and $p=0.0333$, respectively), and treatment uptakes $(p=0.0173$ and $p=0.0150$, respectively) (Table 1). Moreover, among HCV-positive individuals, a lower proportion of females compared with males received treatment ( $\chi^{2}$ test: $p=0.03$ ).

\section{How often hepatitis virus-positive individuals consulted a hepatologist}

No significant relation was observed between the frequency of consultations and the demographic characteristics of hepatitis virus-positive individuals.
Why hepatitis virus-positive individuals did not-consult a hepatologist, access outpatient care regularly, or received treatments

Regarding the reasons that HBV-positive individuals gave for not consulting a hepatologist, "Too busy or no time" was an especially common response among the middle-aged (40-59 years) group $(p=0.009)$ (Supplementary Table S4). However, no significant associations between their demographic characteristics and answers were seen among HCVpositive individuals (Supplementary Table S5). Regarding the reasons for not accessing outpatient care regularly, no significant relation with demographic characteristics was observed among both HBV- and HCV-positive individuals (Supplementary Tables S6 and S7). Regarding the reasons why hepatitis virus-positive individuals received treatment, middle-aged (40-59 years) HBV-positive individuals were likely to answer that "Medical expenses are covered by subsidies" ( $p=0.02)$ (Supplementary Table S8). Moreover, males were more likely than females to answer "Feel the necessity to receive treatment" $(p=0.01)$ (Supplementary Table S8). Additionally, among HCV-positive individuals, males were more likely than females to answer that "Family and friends recommend treatment" $(p=0.008)$ (Supplementary Table S9).

\section{Discussion}

Our intervention successfully improved care-seeking behaviors of hepatitis virus-positive individuals (Figs. 3a, 4a and 5a). In particular, immense improvement was observed in the proportion of individuals who had a consultation in the post-intervention period (Fig. 3a, Supplementary Fig. S3a, S3b and S3c). In most previous studies, patient-navigators have worked on encouraging LTC and the effectiveness of their interventions was confirmed [7, 9, 15-17]. On the other hand, in the USA and the Netherlands, LTC was encouraged through postal mails; these strategies also succeeded in promoting LTC $[8,18]$. Similar to these previous studies, our paper-based intervention involving encouragement by notification letters and brochures was effective in improving LTC. Especially, our annual intervention contributed to continuous increases in the proportions of individuals engaging in care-seeking behaviors. Moreover, numerous previous studies have reported that a lack of human resources hinders the feasibility and sustainability of intervention systems [6, $7,19]$. To counter this problem, a combined use of paper media and human resources could be a practical solution.

The improvement of care-seeking behaviors might also have been induced by the introduction of interferon-free direct acting antivirals (DAAs) since 2014 [4]. However, the proportions of individuals engaged in care-seeking 


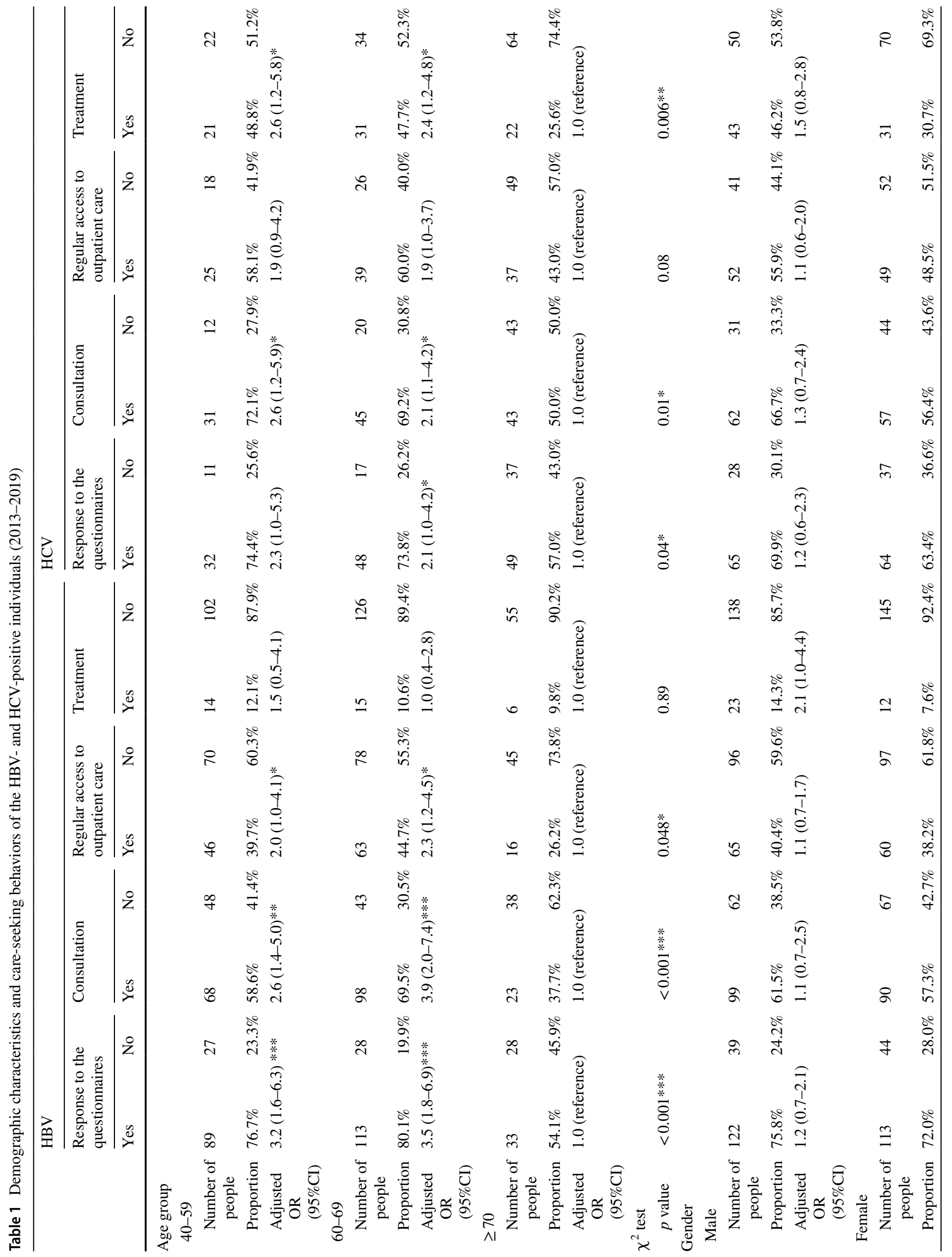




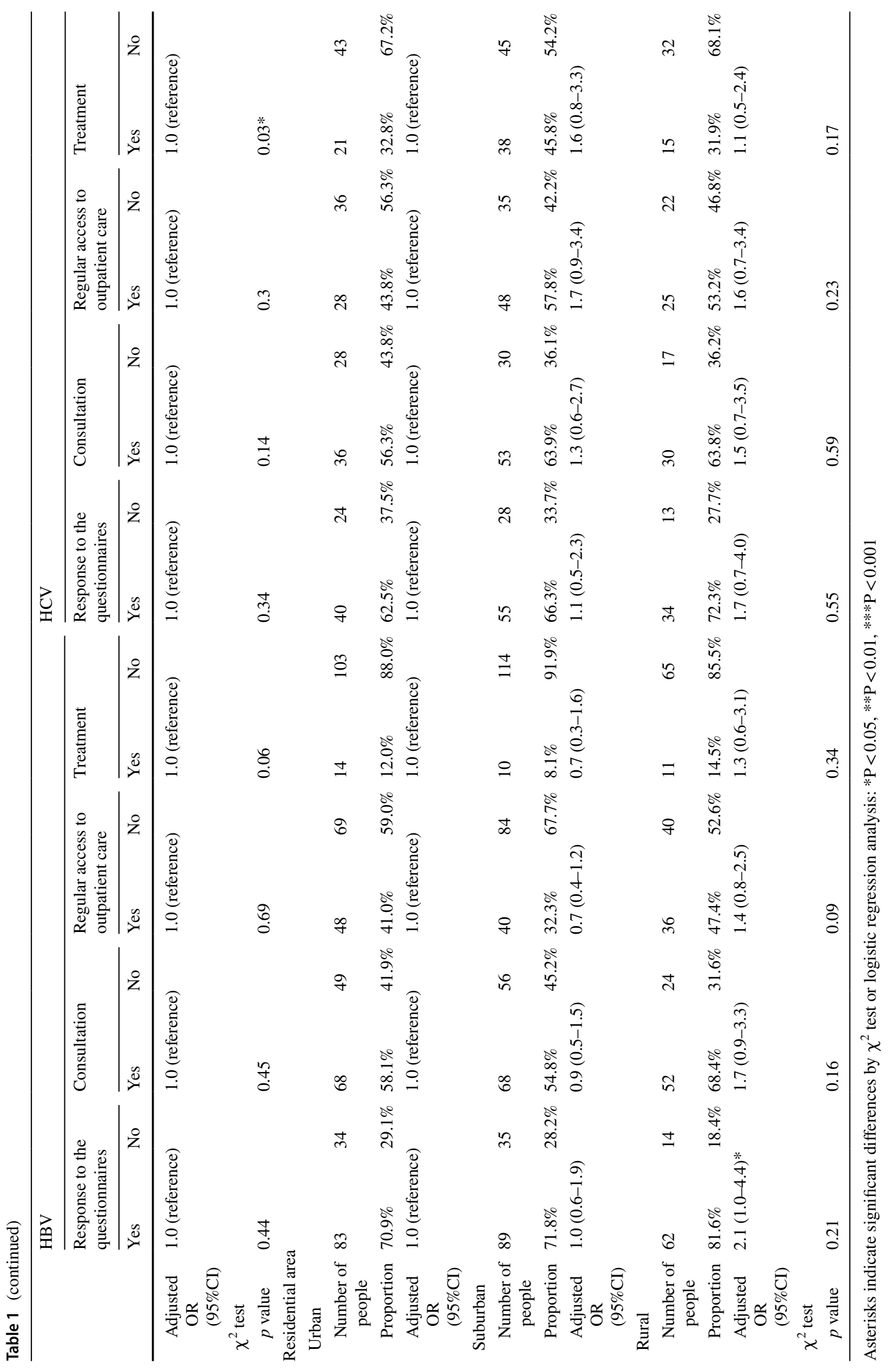


behaviors continued to increase gradually (Figs. 3a, 4a and $5 \mathrm{a})$, not specifically after the introduction of new medicines, which suggests that the incremental improvements were not caused by advances in medicine only.

The timing of enrollment in the intervention system was crucial, as more than $90 \%$ of individuals had consultations within 3 years (Fig. 3c). These results suggest that the encouragement of LTC is more effective for individuals soon after they receive hepatitis virus-positive results. Some previous studies have reported similar results $[13,16$, 18]. Moreover, these results support the radical increase of the proportion of individuals who consulted a hepatologist after the first intervention in 2012 compared to that in the pre-investigation period (Fig. 3a, Supplementary Fig. S3a, $\mathrm{S} 3 \mathrm{~b}$ and S3c). Hence, caregivers should focus their attention on the period immediately following the disclosure of test results.

The individuals who did not consult a hepatologist mostly gave the reason of "No symptoms" (Fig. 3d). These results suggest the need to emphasize the fact that although viral hepatitis is asymptomatic in many cases, it actually requires consultations and attentive care. Moreover, "Too busy or no time" was a frequent reason given by middle-aged individuals for not consulting a hepatologist (Supplementary Table S4): the similar result was observed in Taiwan [20]. This result implies the need for effective systems that accommodate individuals and provide sufficient time for consultations in the modern busy era.

By encouraging outpatient visits, patients with hepatitis are more likely to acquire the habit of accessing outpatient care once they have consulted a hepatologist (Fig. 4b). Nonetheless, some individuals did not access outpatient care for the reported reason of, particularly, "My physician did not recommend accessing outpatient care" (Fig. 4c). This result suggests that advice from a physician has a strong impact on the care-seeking behaviors of hepatitis virus-positive individuals; therefore, knowledge of current treatment and management strategies for viral hepatitis, including LTC strategies, should be kept up-to-date among physicians [6-8, $13,18]$, since these have evolved substantially over the past decade [21].

The treatment uptake was improved post-intervention (Fig. 5a). However, the proportions might still have been low, considering that hepatologists determine eligibility according to the patient's condition, so not all individuals require treatment. Difficulties in increasing viral hepatitis treatment uptake have also been described in previous studies $[13,15]$. As medical science progresses, highly effective drugs, such as nucleic acid analogues and DAAs, have become available for HBV and HCV-infected patients, respectively [22, 23]. In particular, all-oral DAA therapy has been proven to have high efficacy (cure rate $>95 \%$ ) and safety, to be well tolerated, and to have low adverse effects. Given these advantages, more continuous effort is still required to improve treatment uptake. Furthermore, our results showed that hepatitis virus-positive females were less likely to be treated (Table 1). This tendency was also shown in the responses regarding the reasons for receiving treatment (Supplementary Tables S8 and S9). A low prevalence of treatment among females has also been observed in other countries [13, 16], which suggests differences in the influence of interventions and motivation toward treatment between males and females. Since the same HBV and HCV treatments are provided for both genders [22, 23], additional encouragement for treatments among females may be needed.

Despite the repeated intervention, hepatitis virus-positive individuals who were above 70 years of age were still less likely to engage in care-seeking behaviors (Table 1). Adversely, the proportion of older adults among hepatitisvirus positive individuals has been increasing annually (Figs. 2b, c), and HBV- and HCV-positive rates were higher in older generations (Supplementary Figs. S2a and S2b). These results are consistent with those in a previous study, indicating the high prevalence of hepatitis virus infection among older adults in Japan [4]. However, the application of treatment for older hepatitis virus-positive individuals remains controversial [24, 25], so an appropriate medical care and follow-up program needs to be developed.

The scheme of this intervention system has been shared among public health centers in Japan and a detailed manual is available via the Internet [26].

Our study has some limitations. First, the mean response rate for the questionnaires during 2012-2019 was 46.5\% for $\mathrm{HBV}$ and $44.1 \%$ for HCV infection. Meanwhile, throughout the 8 years of follow-up, approximately $70 \%$ of the individuals responded to the questionnaire at least once. Obtaining higher response rate each year would be preferable; however, it should be kept in mind these interventions require careful consideration in terms of social stigma [27, 28]. The considerable endeavor by the Okazaki city public health center was implied from the high response rate over the long-term, despite these difficulties.

Second, a pre-/post-intervention study was conducted instead of a randomized, controlled study since all hepatitis virus-positive individuals should be included in an intervention system from an ethical perspective; the status of hepatitis virus-positive individuals detected in 2008-2012 in the pre-intervention period was regarded as the non-intervention control, and this was compared with that of individuals in the post-intervention period. Although this study design is not ideal, notable increments in the proportions of individuals who were engaging in care-seeking behaviors were observed after the intervention.

Moreover, we performed ITS analysis to evaluate the effect of the intervention. Although ITS is a statistical 
method for evaluating "the slope before the intervention phase", "the level change between phases before/after the intervention", and "the slope after the intervention phase", we have data for only one time point before the intervention in this study. Therefore, we cannot evaluate "the slope before the intervention". "The level change between phases before/ after the intervention" is just the difference between the preinvestigation rate and the pre-investigation rate estimated by linear fitting of the "after intervention" data. Although this is a limitation of the analysis, we were able to show a significant "level change" for "Consultation-HBV", "ConsultationHCV", and "Treatment-HCV".

Furthermore, only three demographic characteristics, age, gender, and residential area, could be analyzed in this study. Management of the personal information of hepatitis virus-positive individuals requires extra attention [29], considering the social stigma of viral hepatitis, and it still might affect their life events in Japan [27, 28]. Under the thorough supervision of the public health center, only anonymous data were used in the present study. Inclusion of their personal factors, such as income, occupation, educational attainment, and their health insurance in the analysis would provide further characteristics of the individuals, but these data should be handled with extreme caution.

Finally, modifications in care-seeking behaviors were analyzed based on a self-reported viral hepatitis care continuum. Therefore, the results are potentially biased and it was difficult to prove the precision of the answers. In addition, the eligibility for treatment among the hepatitis viruspositive individuals could not be confirmed. A scheme for confirming the self-reported viral hepatitis care continuum in public health centers cooperating with hepatologists, needs to be developed in the future.

\section{Conclusions}

Our paper-based reiterative intervention and follow-up system that encourages LTC through cooperation between community health workers, medical experts, and researchers successfully promoted care-seeking behaviors among hepatitis virus-positive individuals. The introduction of specific encouragement modes according to patients' demographic characteristics could improve the system. Especially, innovative intervention methods for older adults are required. In addition, given the high efficacy of current treatments for both HBV and HCV infections, encouragement of treatment uptake should be strengthened through this system. Moreover, additional opportunities for learning further viral hepatitis knowledge among both hepatitis virus-positive individuals and health-care workers, including physicians, and for the development of human resources among hepatitis medical care coordinators, would advance this system. This system could be expected to enhance LTC and eventually contribute to the elimination of viral hepatitis.

Supplementary Information The online version contains supplementary material available at https://doi.org/10.1007/s12072-021-10269-5.

Acknowledgements The authors would like to thank the staff at the Okazaki City Public Health Center for their great assistance with this project. We also would like to thank D. T. Miyamura for the critical discussions and D. Y. Hirama for the technical assistance.

Author contributions HA conceptualized and designed the study. MK conducted the data analysis, interpreted the findings, and drafted the original manuscript under supervision of MS and HA. SH contributed to the communication with hepatitis virus-positive individuals and organized their anonymous data. NK and KY provided medical and technical advice for the project. JT supported and supervised the statistical analysis. HA, KW, KW, MM, and TW participated in the discussion and critical review of the manuscript. All authors contributed to revisions and approved the final manuscript.

Funding The Ministry of Health, Labour and Welfare of Japan provided funding to Hideki Aizaki (Grant Nos. 19HC1001, 20HC1001, and 21HC2001). The Ministry of Education, Culture, Sports, Science and Technology of Japan provided funding to Hideki Aizaki (Grant No. 21K07974). The Advanced Research \& Development Programs for Medical Innovation (AMED, AMED-CREST) provided funding to Hideki Aizaki (Grant Nos. 21fk0210066j0002, 21fk0310112j0705, 21fk0210047h0003, and 21fk0210086j0301).

Availability of data and material Not appreciable.

Code availability Microsoft Excel (Microsoft Japan Co., Ltd., Tokyo, Japan), JMP15 (SAS Institute Japan Ltd., Tokyo, Japan) and R programming language [30] were used.

\section{Declarations}

Conflicts of interest Naoto Kawabe has received research grants from AbbVie GK. Minami Kikuchi, Motoji Sawabe, Haruyo Aoyagi, Kosho Wakae, Koichi Watashi, Satoru Hattori, Kentaro Yoshioka, Junko Tanaka, Masamichi Muramatsu, Takaji Wakita and Hideki Aizaki have no conflict of interest.

Ethical approval The personal information of the hepatitis virus-positive individuals, including age, gender, and residential area, was fully managed by the public health center, only anonymous secondary data were used for the analysis, and the results were shared only by the NIID and regional core center. Written informed consent was obtained from each patient included in the study and the study protocol conforms to the ethical guidelines of the 1975 Declaration of Helsinki. This study was approved by the NIID ethics committee (Approval No.: 796).

Consent to participate All participants provided written informed consent before any study procedures and agreed to receive interventions.

Consent for publication All authors of this manuscript have read and approved the final submitted version and are aware that they are listed as an author on this paper. 


\section{References}

1. WHO. Global Hepatitis Report, 2017. https://apps.who.int/iris/ bitstream/handle/10665/255016/9789241565455-eng.pdf;jsess ionid $=5983$ F3B2F4C7096F427CAE65DA790483 sequence $=1$. Accessed 2 Apr 2021.

2. WHO. Combating Hepatitis B and C to Reach Elimination by 2030. Advocacy brief. https://apps.who.int/iris/bitstream/handle/10665/206453/WHO_HIV_2016.04_eng.pdf? sequence=1. Accessed 2 Apr 2021.

3. Tanaka J, Akita T, Ohisa M, Sakamune K, Ko K, Uchida S, et al. Trends in the total numbers of HBV and HCV carriers in Japan from 2000 to 2011. J Viral Hepat. 2018;25(4):363-72

4. Tanaka J, Akita T, Ko K, Miura Y, Satake M, Epidemiological Research Group on Viral H, et al. Countermeasures against viral hepatitis B and C in Japan: an epidemiological point of view. Hepatol Res. 2019;49(9):990-1002

5. Oza N, Isoda H, Ono T, Kanto T. Current activities and future directions of comprehensive hepatitis control measures in Japan: the supportive role of the Hepatitis Information Center in building a solid foundation. Hepatol Res. 2017;47(6):487-96

6. Cohen C, Caballero J, Martin M, Weerasinghe I, Ninde M, Block J. Eradication of hepatitis B: a nationwide community coalition approach to improving vaccination, screening, and linkage to care. J Community Health. 2013;38(5):799-804

7. Ortiz E, Scanlon B, Mullens A, Durham J. Effectiveness of Interventions for Hepatitis B and C: a systematic review of Vaccination, Screening, Health Promotion and Linkage to Care within Higher Income Countries. J Community Health. 2020;45(1):201-18

8. Chao SD, Chang ET, Le PV, Prapong W, Kiernan M, So SK. The Jade Ribbon Campaign: a model program for community outreach and education to prevent liver cancer in Asian Americans. J Immigr Minor Health. 2009;11(4):281-90

9. Ramirez G, Cabral R, Patterson M, Schoenbachler BT, Bedell D, Smith BD, et al. Early Identification and Linkage to Care for People with Chronic HBV and HCV Infection: The HepTLC Initiative. Public Health Rep. 2016;131(Suppl 2):5-11

10. Denniston MM, Klevens RM, McQuillan GM, Jiles RB. Awareness of infection, knowledge of hepatitis $\mathrm{C}$, and medical followup among individuals testing positive for hepatitis C: National Health and Nutrition Examination Survey 2001-2008. Hepatology. 2012;55(6):1652-61

11. Hyun CS, Ko O, Lee S, McMenamin J. Long term outcome of a community-based hepatitis B awareness campaign: eight-year follow-up on linkage to care (LTC) in HBV infected individuals. BMC Infect Dis. 2019;19(1):638

12. Tait JM, McIntyre PG, McLeod S, Nathwani D, Dillon JF. The impact of a managed care network on attendance, follow-up and treatment at a hepatitis $\mathrm{C}$ specialist centre. J Viral Hepat. 2010;17(10):698-704

13. Vu VD, Do A, Nguyen NH, Kim LH, Trinh HN, Nguyen HA, et al. Long-term follow-up and suboptimal treatment rates of treatment-eligible chronic hepatitis B patients in diverse practice settings: a gap in linkage to care. BMJ Open Gastroenterol. 2015;2(1):e000060

14. Wagner AK, Soumerai SB, Zhang F, Ross-Degnan D. Segmented regression analysis of interrupted time series studies in medication use research. J Clin Pharm Ther. 2002;27(4):299-309

15. Coyle C, Moorman AC, Bartholomew T, Klein G, Kwakwa H, Mehta SH, et al. The Hepatitis C Virus Care Continuum: Linkage to Hepatitis $\mathrm{C}$ Virus Care and Treatment Among Patients at an Urban Health Network, Philadelphia. PA Hepatology. 2019;70(2):476-86
16. Harris AM, Schoenbachler BT, Ramirez G, Vellozzi C, Beckett GA. Testing and Linking Foreign-Born People with Chronic Hepatitis B Virus Infection to Care at Nine U.S. Programs, 2012 2014. Public Health Rep. 2016;131(Suppl 2):20-8

17. Zhou K, Fitzpatrick T, Walsh N, Kim JY, Chou R, Lackey M, et al. Interventions to optimise the care continuum for chronic viral hepatitis: a systematic review and meta-analyses. Lancet Infect Dis. 2016;16(12):1409-22

18. Mostert MC, Richardus JH, de Man RA. Referral of chronic hepatitis B patients from primary to specialist care: making a simple guideline work. J Hepatol. 2004;41(6):1026-30

19. Abara WE, Qaseem A, Schillie S, McMahon BJ, Harris AM, High Value Care Task Force of the American College of P, et al. Hepatitis B Vaccination, Screening, and Linkage to Care: Best Practice Advice From the American College of Physicians and the Centers for Disease Control and Prevention. Ann Intern Med. 2017;167(11):794-804

20. Lin C, Clark R, Tu P, Tu R, Hsu YJ, Nien HC. The disconnect in hepatitis screening: participation rates, awareness of infection status, and treatment-seeking behavior. J Glob Health. 2019;9(1):010426

21. Do A, Reau NS. Chronic Viral Hepatitis: Current Management and Future Directions. Hepatol Commun. 2020;4(3):329-41

22. Ghany MG, Morgan TR, Panel A-IHCG. Hepatitis C Guidance 2019 Update: American Association for the Study of Liver Diseases-Infectious Diseases Society of America Recommendations for Testing, Managing, and Treating Hepatitis C Virus Infection. Hepatology. 2020;71(2):686-721.

23. Terrault NA, Lok ASF, McMahon BJ, Chang KM, Hwang JP, Jonas MM, et al. Update on prevention, diagnosis, and treatment of chronic hepatitis B: AASLD 2018 hepatitis B guidance. Hepatology. 2018;67(4):1560-99

24. Loustaud-Ratti V, Jacques J, Debette-Gratien M, Carrier P. Hepatitis B and elders: an underestimated issue. Hepatol Res. 2016;46(1):22-8

25. Mohammed Abdul MK, Snyder HS, Chunduru M, Lee SMK, Satapathy SK. Hepatitis C Virus in the Elderly in the Direct-Acting Antiviral Era: from Diagnosis to Cure. Curr Treat Options Infect Dis. 2020:1-14.

26. Aizaki H. The manul of follow-up for hepatitis virus-positive individuals ver.3. 2015 (In Japanese) http://www.phcd.jp/02/kenkyu/ sonota/html/KS_kanen.html. Accesed 14 May 2021.

27. Dowsett LE, Coward S, Lorenzetti DL, MacKean G, Clement F. Living with Hepatitis $C$ Virus: a systematic review and narrative synthesis of qualitative literature. Can J Gastroenterol Hepatol. 2017;2017:3268650

28. Smith-Palmer J, Cerri K, Sbarigia U, Chan EKH, Pollock RF, Valentine WJ, et al. Impact of Stigma on people living with chronic Hepatitis B. Patient Relat Outcome Meas. 2020;11:95-107

29. Centers for Disease Control and Prevention. Guidelines For Viral Hepatitis Surveillance And Case Management. https://www.cdc. gov/hepatitis/statistics/surveillanceguidelines.htm. Accessed 2 Aug 2021.

30. R Core Team (2021). R: A language and environment for statistical computing. R Foundation for Statistical Computing V, Austria. URL https://www.R-project.org/.

Publisher's Note Springer Nature remains neutral with regard to jurisdictional claims in published maps and institutional affiliations. 